\title{
Chorégraphie / Philosophie : un dialogue contemporain, entre Deleuze et Le Roy, Salamon, Baehr, Dominguez
}

À propos de Petra Sabisch, Choreographing Relations. Practical Philosophy and Contemporary Choreography, epodium Verlag, München, 2011, 279 pages

\section{Michel Briand}

\section{OpenEdition}

\section{Journals}

Édition électronique

URL : http://journals.openedition.org/danse/703

DOI : $10.4000 /$ danse. 703

ISSN : 2275-2293

Éditeur

ACD - Association des Chercheurs en Danse

Référence électronique

Michel Briand, «Chorégraphie / Philosophie : un dialogue contemporain, entre Deleuze et Le Roy, Salamon, Baehr, Dominguez », Recherches en danse [En ligne], Actualités de la recherche, mis en ligne le 24 avril 2014, consulté le 19 septembre 2020. URL : http://journals.openedition.org/danse/703 ; DOI : https://doi.org/10.4000/danse.703

Ce document a été généré automatiquement le 19 septembre 2020 


\section{Chorégraphie / Philosophie : un dialogue contemporain, entre Deleuze et Le Roy, Salamon, Baehr, Dominguez}

À propos de Petra Sabisch, Choreographing Relations. Practical Philosophy and Contemporary Choreography, epodium Verlag, München, 2011, 279 pages

Michel Briand

\section{RÉFÉRENCE}

Petra Sabisch, Choreographing Relations. Practical Philosophy and Contemporary Choreography, epodium Verlag, München, 2011, 279 pages 
1 La danse et la philosophie, théorique et pratique, sous leurs aspects conjointement esthétiques, éthiques, politiques, épistémologiques, dialoguent avec constance, de certains penseurs antiques, tels Platon et Plutarque, aux études récentes d'Alain Badiou ou de Giorgio Agamben en passant par Nietzsche ou encore Valéry. Et l'on dit volontiers qu'une pièce chorégraphique donne à penser, voire est «bonne à penser ", ou, réciproquement, qu'une pensée, un style d'écriture, voire un mode d'argumentation relèvent, au moins par métaphore, d'une sorte de chorégraphie. Mais ce parallèle liminaire, superficiel, reste toujours à nuancer et troubler, tout en l'ouvrant à de nouveaux enjeux,

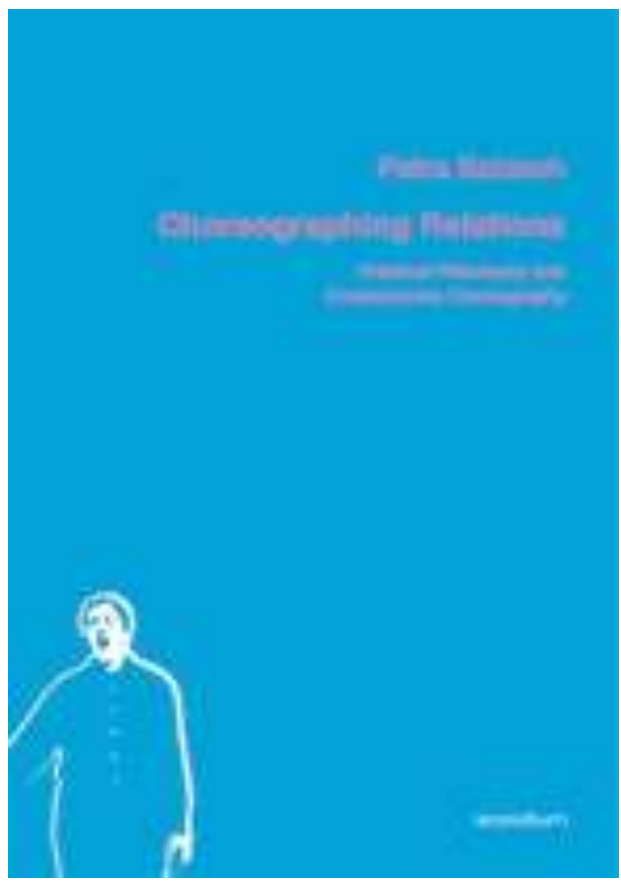
comme le font par exemple Véronique Fabbri (Danse et philosophie. Une pensée en construction, L'Harmattan, 2007) et Frédéric Pouillaude (Le désoeuvrement chorégraphique. Étude sur la notion d'œuvre en danse, Vrin, 2009). Ces deux exemples montrent la variété possible des approches, la première insistant, d'une manière sous certains aspects similaire à ce qui se passe dans l'ouvrage ici recensé, sur le dialogue privilégié de la danse avec l'architecture et les concepts de construction - destruction déconstruction, en référence notamment à Valéry, Benjamin et Derrida; le second proposant, dans une perspective transhistorique, après avoir établi le constat de l' absence d'œuvre en danse, un nouveau concept, le désoeuvrement, propre à caractériser la création chorégraphique, dans sa fragilité même.

2 Par ailleurs, on peut penser qu'une réflexion philosophique vivante est foncièrement artistique (créatrice) et en retour que les chorégraphies que la critique contribue à mieux observer et ressentir mettent en jeu de véritables questions philosophiques. Cette relation particulière de l'art à la philosophie et de la philosophie à l'art, qui n'est pas du tout la simple application d'une "grille de lecture " conceptuelle à des objets esthétiques conçus comme clos, univoques et peu réflexifs, se développe tout particulièrement dans les approches de type phénoménologique, par exemple telles qu'elles sont étudiées et pratiquées ici même dans les contributions de Paule Gioffredi sur Myriam Gourfink et Merleau-Ponty ou de Katharina Van Dyk sur Laurence Louppe, la kinesphère et le pré-mouvement ${ }^{1}$.

Et dans son ouvrage issu d'une thèse de doctorat soutenue en 2010, dont le titre désigne plutôt un processus que le produit d'un processus (Choreographing Relations), Petra Sabisch, elle-même à la fois chorégraphe, performeuse et philosophe (spécialiste d'esthétique et de philosophie pratique), propose une réflexion ample et minutieuse sur les liens d'une certaine danse, disons provisoirement "méta-danse " ou "danse critique ", et d'une certaine pensée, en l'occurrence surtout l'empirisme transcendantal comme méthode, tel que l'ont construit Gilles Deleuze et Félix Guattari. Au-delà de la vogue internationale que connaissent de multiples concepts issus de la French Theory ${ }^{2}$, 
aussi dans le domaine des arts du spectacle vivant (ou plus justement ici des performance studies), vogue qui pourrait rester un plaquage abstrait du théorique sur le pratique, on ne peut qu'être sensible à l'intrication dynamique de deux modes d'accès, à dominante conceptuelle et gestuelle, au monde, au corps, au temps ou à l'espace, donc au rythme et au "spectacle " ou à la "performance ", qui sont ici mis en scène et en question. Le propos de l'introduction, réaffirmé par l'ouverture (Opening - in vivo) du premier chapitre (intitulé Contamination), présente un programme riche, très documenté, qui semble ensuite rempli : plus que de concepts, il s'agit précisément de «mouvements-pensées pratiques» (practical movements-thoughts), dont l'étude doit permettre d'associer efficacement les approches soit expérimentales soit conceptuelles de la danse. Enfin, le corps, en particulier le corps dansant, ne s'y définit pas comme un donné compositionnel, organiciste, mais comme une résultante et une puissance capable d'affecter et d'être affecté, selon la référence spinoziste de Deleuze et Guattari, citant le « corps sans organes » artaudien ${ }^{3}$.

Petra Sabisch s'intéresse en effet d'abord à la façon dont des chorégraphies contemporaines, non mimétiques (arepresentational) mais expérimentales, interrogent et reformulent un matériau à la fois cognitif et matériel: entre procédures chorégraphiques et méthodes d'observation critique, souvent d'inspiration phénoménologique, il y a une analogie et une complémentarité essentielles, qu'une création et une réception vraiment actives peuvent faire agir et vivre, mais aussi une toujours possible et fluide rencontre (encounter), qui trouble tout en les gardant à l'esprit les différences entre théorie et pratique. Et ce mode de rencontre est revendiqué comme typique d'un air du temps contemporain (at the turn of the twentieth century). Ici se situe d'ailleurs l'une des faiblesses possibles du propos, empreint de "présentisme", sur une question qui n'est pas vraiment nouvelle, alors qu'elle est présentée comme intrinsèquement typique de pièces créées entre 1998 et 2002, au tournant du siècle donc: d'autres moments, y compris vraiment plus anciens, de l'histoire de la danse, autour des ballets baroque ou romantique ou encore de l'expressionnisme, par exemple, ont aussi vivement interrogé et mis en scène cette rencontre sensible entre philosophie pratique et chorégraphie. Mais c'est aussi l'une des forces de l'ouvrage, puisque le rapport entre danse et philosophie n'est pas établi sur des bases thématiques ou formelles, mais s'opère par le biais de la méthode comme procédure commune (encounter in method) aux sciences humaines et à la performance et comme pratique singulière (material and experimental practice). Le terme crucial est celui de relations ${ }^{4}$, comme l'indique le titre, complexe, comme entre "chorégraphier des relations " ("mettre en danse des relations») et "des relations chorégraphisantes " ( « des relations qui écrivent / créent de la danse »?). Et le style même de l'ouvrage et sa composition, en cela aussi procédurale que les œuvres orchestiques ou philosophiques qu'elle étudie ou dont elle joue, montrent avec constance cette alliance entre pensée et corps, sensation et cognition, ou encore création et réception. Dès le second sous-titre, en page intérieure, penseurs et chorégraphes sont présentés sur le même plan, en ordre alphabétique: in the works of Antonia Baehr, Gilles Deleuze, Juan Dominguez, Félix Guattari, Xavier Le Roy and Eszter Salamon (p. 3).

5 Cinq pièces de danse sont abordées, et d'abord, dans la première moitié du volume, la désormais célèbre proposition de Xavier Le Roy, la conférence - performance Product of Circumstances (1999), mise en relation avec le concept de Contamination (p. 17-93) puis avec celui d'Articulation (p. 95-143). Ces deux concepts sont signifiés par deux noms d'action, susceptibles d'aider le critique, le créateur / performeur et le spectateur à 
comprendre ce que "peut faire la / une chorégraphie » (What can choreography do), qui donc, selon la perspective de l'auteure, contamine et articule. La contamination concerne les transformations qualitatives dont sont capables des corps, par leur capacité à (se) réunir, (s')assembler ou (s')agencer (power to assemble), en particulier dans la pièce de Xavier Le Roy, mise en scène autobiographique et réflexive, en même temps que performative, du trajet par lequel l'artiste s'est déplacé de la biologie moléculaire (oncologique) à la danse dite contemporaine, d'un travail de production scientifique à un travail de création artistique, assemblés dans / par une chorégraphie. Ce mouvement réciproque, entre médecine et danse, qui fait de la pièce un assemblage en mouvement, conjuguant jeux d'adaptation, hybridation et altérité, rejoint la question initiale des relations entre chorégraphie et philosophie. Et concrètement, l'analyse se concentre sur les circonstances scéniques de la production chorégraphique, la lumière, la construction des espaces, la relation du performeur avec le public, en termes épistémiques et affectifs, etc.

6 Petra Sabisch donne ainsi une application pratique de l'« empirisme transcendantal» deleuzien (transcendental empiricism) ${ }^{5}$, en tant qu' « épistémologie de la rencontre », qui fait l'objet du chapitre 1.3, à visée d'abord méthodologique : l'ensemble de la réflexion deleuzienne y est traitée, souvent en opposition avec Derrida, en particulier les concepts et références applicables aux études de danse, comme le devenir, le corps sans organes, le pli, la différence et la répétition, l'immanence, les analyses relatives à Hume et Kant, à la peinture de Bacon, au cinéma. Puis l'articulation (traduction de l'allemand Gliederung, de gliedern « organiser, structurer »), au long du chapitre 2, paradoxalement plus abstrait, à visée épistémologique, et fondé sur un jeu subtil entre notions quasihomonymes (to make sense, sensible), concerne les transformations qualitatives du sens / sentiment (sense), dans une perspective selon laquelle la chorégraphie a quelque chose d'un langage, doublement articulé, en termes de composition comme de différentiation. La pensée philosophique et la performance chorégraphique se retrouvent à niveau, en dialogue "sensible $»^{6}$, quand les signes que l'une et l'autre emploient sont de type encore deleuzien, non saussurien, référentiel ou représentationnel, mais lié aux notions de différence et d'intensité, comme dans Proust et les signes, 1964, et Différence et répétition, 1968. Il ne s'agit pas de sémantique mais de sémiologie, et d'une "signification" fondée sur l'interaction différentielle de mouvements, actions physiques et langagières, structurations temporelles et spatiales, conventions spectaculaires et culturelles, selon notamment les analyses présentées dans Logique du sens, 1969, Logique de la sensation, 1981, ou L'image-mouvement, 1983, et L'image-temps, 1985. La pièce Product of Circumstances, aussi en relation avec des pièces antérieures qu'elle cite (Things I hate to admit et Burke, de 1997), devient l'exemple privilégié d'une nouvelle "topologie des articulations", dont la signification résulte d'un processus fondé sur un jeu de compositions machiniques et d'assemblages de signes différentiels, propres à transformer le sensible, plus qu'à le représenter.

7 Le troisième et dernier chapitre de l'étude, qui se présente en partie comme un ensemble dialectique ternaire, Choreographing Relations of Contamination and Articulation (p. 145-234), se focalise successivement sur quatre pièces chorégraphiques et deux notions encore issues d'une "boîte à outils » critique d'inspiration deleuzienne, le retenu et le dé-lire. Le chapitre Retenu (p.145-208) étudie Self Unfinished (1998), encore de Xavier Le Roy ${ }^{7}$, et What a body you have, honey (2001) de Eszter Salamon ${ }^{8}$, dans la perspective du « retenu cinématique » (" cinematic Retenu »), puis Holding Hands (2001) d'Antonia Baehr, par le « retenu cinémotionnel » (« cine-emotional Retenu »). Le terme 
retenu, à la fois associé à des linogravures de Matisse (Le Retenu et Le lancé, 1939) et aux idées de Cunningham sur la "forme du temps » (shape of time) et la forme temporelle du mouvement, désigne un régime esthétique, d'ordre foncièrement kinesthésique, dans lequel mouvement et perception sont indissociablement liés à des modalités temporelles comme la rétention husserlienne et la durée bergsonienne. Le retenu cinématique, encore en référence à Deleuze, est exemplifié par la seconde pièce de Xavier Le Roy étudiée ici et par celle d'Eszter Salamon, où sont observées surtout les transformations corporelles qualitatives d'origine kinétique. Par l'analyse détaillée de Self Unfinished, en tant que dispositif construisant un temps immanent spécifique, en collaboration avec le vidéaste Laurent Goldring, puis par l'étude non moins précise de la pièce d'Eszter Salamon, dont le régime esthétique, qui travaille les transformations d'un corps dans la durée, en transposant l'image cinématographique en chorégraphie cinématique, Petra Sabisch démontre combien l'idée (binaire) de «non-danse », n'est pas opératoire, surtout pour ces pièces.

8 Dans la proposition d'Antonia Baehr, dont une photographie des deux performeurs (Antonia Baehr et William Wheeler) se tenant la main a servi, non sans humour, pour la couverture de l'ouvrage, le retenu cinémotionnel permet, quant à lui, de rendre compte des transformations qualitatives des sensations, en mode continu. La pragmatique proposée travaille le rapport entre émotions et altérations corporelles, dans une composition de type plus musical et physique que théâtral, qui vise à " défacialiser " (defacialize) les émotions, en les fondant sur des sensations et mouvements du corps même et en présentant surtout le passage de l'un à l'autre. Et la chorégraphe insiste explicitement, dans une interview conjointe avec Xavier Le Roy, sur le fait que la composition de la pièce provient, au départ, d'une réflexion sur la catharsis, dont elle inverse le lien avec la mimesis aristotélicienne ${ }^{9}$ : la partition remplace le texte dramatique, et la présentation de figures émotionnelles reconnaissables, au lieu de personnages, vise non à l'identification psychologique, mais à la production d'effets kinesthésiques, donc corporels, sur le public.

9 D'autre part, le chapitre Dé-lire (p. 209-234, où le concept de dé-lire est traduit par rereading ${ }^{10}$ ) étudie la pièce All Good Spies are My Age (2002) de Juan Dominguez, comme présentation performative de sa propre "re-lecture ", nécessairement réflexive : trois temporalités simultanées sont explicitement à l'œuvre, celle de la performance, celle de l'image-texte du récit de la performance, projeté en diapositives, et celle, objective et subjective, du temps et de la durée qui soutiennent les deux précédentes. Il s'agit véritablement de "chorégraphier la puissance du temps » (choreographing the force of time), tout en re-lisant (commentant, interprétant, accompagnant?) la production chorégraphique présentée (rereading the production of choreography), en cinq séquences successives que scandent dix coïncidences significatives entre «la vie » et «l'œuvre». Le montage temporel construit par le dispositif vidéographique diffère en cela du retenu cinématographique ou cinémotionnel : la réception active du public ne se fonde pas sur la perception de mouvements mais sur la lecture de textes brefs projetés en diapositives, qui stimulent la pensée et l'imagination d'une pièce dansée fictivement à venir. La notation barthésienne d' "effets de réel » est sans doute opératoire ici ${ }^{11}$, en résonnance avec la «fabulation » bergsonienne et, enfin, encore chez Deleuze, sur la "puissance du faux", en particulier dans l'art de la narration cinématographique qu'étudie L'image-temps (1985). La boucle critique est claire: nous retrouvons ici, dans une perspective temporelle, une partie de ce qui se jouait, sur un plan d'abord spatial, dans la première pièce de Xavier Le Roy, et dans les deux cas, c'est la notion de 
production méta-chorégraphique qui fait sens, en même temps que sensation, avec la critique des circonstances de cette production, sur un mode aussi humoristique que pathétique d'ailleurs, ou encore plus classiquement neutre.

La conclusion (p. 234-245) est une bonne synthèse de ce parcours, par lequel l'auteure montre que les pièces chorégraphiques étudiées définissent un art que fondent des "pratiques kinésiques réfléchies, relationnelles et participatives » (thoughtful, relational and participatory movement-practices, p. 243), opposées, d'un point de vue immanent, à une conception représentationnelle de la danse et à une relation statique et unidimensionnelle du public avec le mouvement qui lui est donné à voir, ressentir et penser. La démonstration est d'autant réussie qu'elle s'achève, p. 245, avec l'espoir de nouvelles "relations participatives", à venir, encore à inventer. Un seul regret: l'ouvrage dans son ensemble se ressent de son premier statut de thèse doctorale, où les effets suggestivement évoqués à propos de toutes les pièces observées n'informent pas vraiment le style et la composition d'une réflexion qui pourrait s'alléger, en signalant par elle-même les noces intelligentes et sensibles, conceptuelles et corporelles, de la danse et de la philosophie. Mais c'est peut-être là une invitation à lire aussi ce texte, un peu comme chez Dominguez, comme le script paradoxalement développé d'une performance possible, à venir.

\section{BIBLIOGRAPHIE}

BOURRIAUD Nicolas, L'Esthétique relationnelle, Dijon, Les Presses du Réel, 1998.

CUSSET François, French Theory : Foucault, Derrida, Deleuze et Cie et les mutations de la vie intellectuelle aux États-Unis, Paris, La Découverte, 2003.

DELEUZE Gilles, Proust et les signes, Paris, PUF, 1964.

DELEUZE Gilles, Différence et répétition, Paris, PUF, Épiméthée, 1968.

DELEUZE Gilles, Logique du sens, Paris, Minuit, 1969.

DELEUZE Gilles,Francis Bacon. Logique de la sensation, Paris, Seuil, L'Ordre philosophique, 1981.

DELEUZE Gilles,Cinéma 1. L'image-mouvement, Paris, Minuit, 1983.

DELEUZE Gilles,Cinéma 2. L'image-temps, Paris, Minuit, 1985.

DELEUZE Gilles \& GUATTARI Félix, Mille Plateaux. Capitalisme et schizophrénie 2, Paris, Minuit, 1980.

FABBRI Véronique, Danse et philosophie. Une pensée en construction, Paris, L'Harmattan, 2007.

MARX William, Le Tombeau d'CEdipe. Pour une tragédie sans tragique, Paris, Minuit, 2012.

PERRIN Julie, Figures de l'attention. Cinq essais sur la spatialité en danse, Dijon, Les presses du réel, 2012.

POUILLAUDE Frédéric, Le désoeuvrement chorégraphique. Étude sur la notion d'œuvre en danse, Paris, Vrin, 2009.

RANCIÈRE Jacques, Le partage du sensible. Esthétique et politique, Paris, La Fabrique, 2000. 
SAUVAGNARGUES Anne, Deleuze, l'empirisme transcendantal, Paris, PUF, Philosophie d'aujourd'hui, 2008.

\section{NOTES}

1. Les articles de Paule Gioffredi et Katharina Van Dyk sont publiés dans Recherches en danse, $\mathrm{n}^{\circ} 1$, 2014.

2. Voir CUSSET François, French Theory: Foucault, Derrida, Deleuze et Cie et les mutations de la vie intellectuelle aux États-Unis, Paris, La Découverte, 2003. Cette généalogie critique indirecte est d'autant plus à signaler que Petra Sabisch s'explique d'ailleurs en fin d'introduction (pp. 15-16) sur les problèmes de la traduction, non seulement de termes français ou allemands en anglais, mais aussi plus largement comme "reconfiguration relationnelle de signification " (selon la formule de Gabriel Rockhill, voir plus bas note 3), liée, en ce qui la concerne, à la créativité chorégraphique remarquable qui s'est développée à Berlin et Paris, au tournant du XXI ${ }^{\mathrm{èm} e}$ siècle. L'auteure, du fait aussi que l'ouvrage est rédigé en anglais, présente souvent Deleuze, par exemple, par sa réception anglophone, ce qui ne manque pas d'influer sur son propre travail de conceptualisation, voire sur sa connaissance des études chorégraphiques contemporaines d'expression française.

3. DELEUZE Gilles, GUATTARI Felix, Mille Plateaux. Capitalisme et schizophrénie 2, Paris, Minuit, «Critique », 1980.

4. Une référence importante est BOURRIAUD Nicolas, L'Esthétique relationnelle, Dijon, Les Presses du Réel, 1998.

5. Voir surtout SAUVAGNARGUES Anne, Deleuze, l'empirisme transcendantal, Paris, PUF, «Philosophie d'aujourd'hui », 2008.

6. En référence aussi aux travaux de Jacques RANCIÈRE, en particulier Le partage du sensible. Esthétique et politique, Paris, La Fabrique, 2000, dans sa traduction anglaise par Gabriel ROCKHILL, qui, en 2006, conserve au terme sensible son sens français, différent de l'anglais courant (« raisonnable»).

7. Cette pièce est étudiée par PERRIN Julie, Figures de l'attention. Cinq essais sur la spatialité en danse, Dijon, Les presses du réel, 2012, pp. 53-94 (Xavier Le Roy, Self-Unfinished ou le bouleversement du visible »), qui cite aussi l'analyse de Petra Sabisch, à propos de la lecture deleuzienne à laquelle nous invite lui-même le chorégraphe.

8. Il est aussi question de la pièce nvsbl (2006).

9. Antonia Baehr rejoint ici, sans doute involontairement, les travaux récents ou non qui montrent que la catharsis aristotélicienne désigne un type d'expérience plus physique que traditionnellement littéraire. Cf. notamment MARX William, «La véritable catharsis aristotélicienne. Pour une lecture philologique et physiologique de la Poétique ", Poétique 166, 2011, repris dans Le Tombeau d'EEdipe. Pour une tragédie sans tragique, Paris, Éditions de Minuit, 2012.

10. Ce sens n'est pas attesté en français, ni étymologiquement (dé-lirer c'est d'abord «sortir du sillon ", la lira en latin, un peu comme dé-railler), ni dans les emplois contemporains. On a là un bon exemple des limites que rencontrent des transferts culturels aussi massifs que celui qu'a connu la French Theory, désormais revenue en Allemagne, puis en France. Cette remarque n'enlève rien à l'analyse menée ici par Petra Sabisch, qui l'intègre seulement dans un contexte critique très particulier, devenu « global » et dominant.

11. Petra Sabisch ne semble pas inscrire l'analyse de Roland Barthes dans une histoire plus longue de la pragmatique rhétorique (et littéraire), à laquelle pourtant il se réfère souvent, à partir par exemple de la notion antique d'enargeia / evidentia, ou à propos de la description. 


\section{RÉSUMÉS}

Petra Sabisch s'inspire principalement de l'empirisme transcendantal deleuzien, appliqué à cinq pièces chorégraphiques des années 1998-2002, créées à Berlin et Paris. Les concepts critiques principaux sont ceux de relation, contamination, articulation, puis retenu et dé-lire.

Petra Sabisch mostly refers to Deleuze's transcendantal empiricism, which she applies to 5 choreographic performances created in the 1998-2002's, in Berlin and Paris. The main critical concepts she uses are relation, contamination, articulation, retenu, and dé-lire.

\section{INDEX}

Mots-clés : empirisme transcendantal, Deleuze (Gilles), philosophie, relation esthétique, Le Roy (Xavier)

Keywords : transcendantal empiricism, Deleuze (Gilles), philosophy, relational esthetics, Le Roy (Xavier)

\section{AUTEURS}

\section{MICHEL BRIAND}

Michel Briand, professeur de langue et littérature grecques (U. de Poitiers). Travaille aussi sur la rémanence de l'Antiquité et sur la danse contemporaine. Derniers ouvrages : comme directeur, La trame et la tableau : poétique et rhétorique du récit et de la description dans l'Antiquité grecque et latine, PU de Rennes, 2013, et comme auteur (commentaire et nouvelle traduction), Pindare. Olympique, Belles Lettres, 2014. Dernier article sur la danse : « Danse - récit (et action) / danse matière (et création) : pour une esthétique comparée de l'antique et du contemporain, d'Homère et Lucien à Gallotta, Chopinot, Duboc, et retour ", Rémy Poignault (dir.), Présence de la danse dans l'Antiquité Présence de l'Antiquité dans la danse, Clermont-Ferrand, 2013, pp. 409-423. 